\title{
In the Heat of the Summer
}

\section{Lessons from the Heat Waves in Paris}

\section{Emmanuelle Cadot, Victor G. Rodwin, and Alfred Spira}

Climate change and human health are intertwined. ${ }^{1}$ The heat waves in Chicago, in 1995, and in Paris, in 2003, followed by Hurricane Katrina's destruction of New Orleans, raised awareness of the risks faced by vulnerable older people. Many cities have responded by announcing emergency preparedness plans; some of these plans have already been tested. Last summer, from July 27 to August 5, New York City suffered a mild heat wave with temperatures reaching $100^{\circ} \mathrm{F}$. Paris, as well, was hit by another heat wave from July 17 to July 29, with maximum temperatures reaching $104^{\circ} \mathrm{F}$, which was considerably milder than in 2003 when they often exceeded $110^{\circ} \mathrm{F}$. In New York, there were 100 "excess deaths," an increase of $8 \%$ over past trends. ${ }^{2}$ In Paris, the number of excess deaths in 2006 (42), also an increase of $8 \%$, was considerably lower than the 1,294 deaths registered in 2003-an increase of $190 \%$ compared to the preceding three-year average.

Given existing surveillance capacity, it is impossible to know whether the reduction in excess deaths in Paris was due, partly, to its enhanced preparedness or whether it reflects no more than the effects of a far milder heat wave. Nevertheless, the milder heat wave of 2006 does provide an opportunity to examine the actual implementation of the heat wave preparedness plan. In light of ongoing efforts to develop such plans in cities worldwide and completed studies on the effects of the 2003 heat wave in Paris, what may be learned to promote urban health and improve understanding of the factors that put vulnerable older people at greatest risk?

\section{THE PARIS HEAT WAVE PREPAREDNESS PLAN}

Approximately 9 months following the catastrophic heat wave of 2003, the Paris Mayor's Office developed a plan to target interventions for those who considered themselves to be most vulnerable. The Mayor wrote a letter to all older Parisians, as well as to all those suffering from severe handicaps and therefore eligible for special services. He invited these 400,000 Parisians to register with the City's Social Services Agency and thereby benefit from direct phone contact and offers of special assistance in the event of another heat wave. In June 2006, about 13,000 people, mostly those 65 years and over, had registered. On July 17th, the plan was activated when the National Weather Institute issued a heat wave warning. Until July 28, the Social Services Agency phoned all registered individuals every other day to check on

Cadot and Spira are with the Atelier Parisien de Santé Publique, Service de Santé Publique et d'Epidémiologie, Hôpital de Bicêtre, Le Kremlin Bicêtre, France; Rodwin is with the Wagner School, New York University and World Cities Project, International Longevity Center, New York, USA.

Correspondence: Emmanuelle Cadot, Atelier Parisien de Santé Publique, Service de Santé Publique et d'Epidémiologie, Hôpital de Bicêtre, 82, rue du Général Leclerc, 94276, Le Kremlin Bicêtre, France.

(E-mail: cadot@vjf.inserm.fr) 
them. When those who registered required assistance, based on a preliminary screening, they were evaluated, once again, by physicians, to determine their health status. Nearly 800 older people were evaluated by a medical team over these 11 days; 200 were called back. Most of the city's interventions were limited to providing information on what to do and where to go during the heat wave. About 30 people were transported to air-conditioned adult day centers and 18 benefited from urgent medical attention.

Because the 2006 heat wave was milder in duration and most meteorological characteristics than the one in 2003, it is difficult to attribute the lower number of excess deaths only to the city's preparedness plan. It seems plausible, however, that the plan increased the number of daily contacts for some socially isolated older people and restored for them - in some small way - a social network. Based on a synthesis of studies about the 2003 heat wave, this is precisely what must be done.

\section{THE AUGUST 2003 HEAT WAVE}

Between August 1 and 20, 2003, a deadly European heat wave resulted in some 14,800 excess deaths in France, an increase of 55\%. ${ }^{3}$ Paris was disproportionately affected (an increase of $190 \%$ ). In contrast to the rest of France, where $65 \%$ of excess mortality afflicted institutionalized older people, in Paris, $74 \%$ of excess deaths occurred among those who were living at home.

It is well-known that Paris has a higher share of people 80 years and over than the rest of France. Also, 66\% of Parisian women in this age cohort live alone, which far exceeds the average for France $(50 \%)$. But neither age, nor gender density, nor temperature levels are sufficient to explain the concentration of excess deaths in Paris compared to the rest of France. Do they reflect the individual characteristics of Parisians or the character of the health care system and the neighborhoods in which they lived? The health system seems an implausible suspect, in light of its high performance in many dimensions. ${ }^{4}$ But since this catastrophe took French physicians and the public health establishment by surprise, it is important to review at least partial answers to these questions, particularly because the evidence has not been widely available in English.

\section{FINDINGS TO DATE}

The most important risk factors for dying during the heat wave of 2003 were being a woman 75 years old and older and living alone at home. ${ }^{5}$ Parisians who were unmarried (and more frequently live alone) were also at higher risk of death than those who lived as couples. Higher risk associated with gender (being a woman), however, did not apply to foreign nationals. Older women, particularly those from Africa and Asia - in contrast to French women - live, more often, in multigenerational families with stronger mutual support and social networks; hence a lower risk of social isolation. As Klinenberg found in his "autopsy" of the 1995 Chicago heat wave, ${ }^{6}$ social isolation is associated with higher risk of mortality. Moreover, mortality rates were highest in the southeastern parts of the city, indicating a shift in the geographic mortality distribution from poorer to more well-to-do neighborhoods, in comparison to the usual mortality gradient across Paris neighborhoods. These findings could reflect temperature variations, as well as socioeconomic and demographic differences among the northeast and southwest arrondissements of Paris. 
Among a sample of vulnerable older people who received a personal autonomy allowance (PAA), based on their level of disability, living alone was the only significant risk factor for excess mortality. Whether PAA beneficiaries were community-dwelling or institutionalized, their disability levels were not significant risk factors. Among older nursing-home residents, Holstein et al. followed a cohort of 4,403 residents from May through December of 2003 and found that women in better health were at higher risk of death than their more vulnerable counterparts. ${ }^{7}$ During the heat wave, there was an increased risk of death for all residents, but the mortality increase was significantly lower for the most dependent residents. Analysis of the weekly distribution of deaths reveals no "harvesting effect"-no compensating reduction in mortality after the heat wave, neither in the final days of August nor in the weeks that followed. Thus, the deceased were not at risk of dying from medical or any other causes in the aftermath of the heat wave.

\section{LESSONS AND DIRECTIONS FOR HEAT-WAVE PREPAREDNESS}

Perhaps the most important lesson of the Paris heat waves is to recognize that preparedness efforts and promotion of urban health must focus not only on individual but also on neighborhood risk factors. The essential components of the heat wave preparedness plan focused on individual risk factors, e.g., encouraging older persons to drink more, offering special services to those who perceived themselves to be in need of assistance, and increasing medical surveillance of these individuals. In the future, more attention should focus on targeting some of the neighborhoods that suffered disproportionate excess mortality.

\section{REFERENCES}

1. Epstein P. Climate change and human health. N Engl J Med. 2005;353:14.

2. NYCDHMH. Deaths Associated with Heat Waves in 2006. New York: NYCDHMH; 2006. Available from: http://www.nyc.gov/html/doh/downloads/pdf/survey/survey2006heatdeaths.pdf.

3. Fouillet A, Rey G, Laurent F, et al. Excess mortality related to the August 2003 heat wave in France. Int Arch Occup Environ Health. 2006;80:16-24.

4. Gusmano MK, Rodwin VG, Weisz D. A new way to compare health systems: avoidable hospital conditions in Manhattan and Paris. Health Aff. 2006;25:510-520.

5. Canouï-Poitrine F, Cadot E, Spira A. Excess deaths during the August 2003 heat wave in Paris, France. Rev Epidemiol Sante Publique. 2006;54:127-135.

6. Klinenberg E. Heat wave. A social autopsy of disaster in Chicago. Chicago: The University of Chicago Press; 2002.

7. Holstein J, Canouï-Poitrine F, Neumann A, Lepage E, Spira A. Were less disabled patients the most affected by 2003 heat wave in nursing homes in Paris, France? J Public Health. 2005;27:359-365. 